\title{
Understanding the enhancement of coating's thermal cracking resistance by multiple segmentation
}

\author{
Xuejun Chen ${ }^{1, a}$ and Guangnan Chen $^{2}$
}

${ }^{1}$ Department of Applied Mechanics, University of Science and Technology Beijing, 30 Xueyuan Road, Beijing 100083, People's Republic of China

${ }^{2}$ Institute of Mechanics, Chinese Academy of Sciences, 15 Beisihuanxi Road, Beijing 100190, People's Republic of China

${ }^{a}$ chenxuejun@ustb.edu.cn

Keywords: Segmented coating; Cracking; Segmentation crack; Thermal Stress Intensity Factor Abstract In this paper, the thermal shock induced cracking behavior of a segmented coating on the outer surface of a hollow cylinder has been investigated. The driving force for the propagation of multiple segmentation crack, represented by the Thermal Stress Intensity Factor (TSIF), was determined by combination of the principle of superposition and the finite element method. The maximum TSIF has been shown to occur neither at the beginning nor at the steady state of thermal transients, but at an intermediate instant. As the spacing between multiple segmentation cracks decreases, the magnitude of TSIF first plateaus, and then decreases sharply. This quantitative mechanistic result rationalizes the experimental observations that a segmented coating can exhibit much higher thermal shock resistance than an intact counterpart, if only the segmentation crack spacing is narrow enough. Some other parameters affecting TSIF, such as segmentation crack depth and convection severity, were also discussed.

\section{Introduction}

Protective coatings have been widely used in thermal environments to improve certain surface properties of the substrate, e.g., thermal, corrosion and wear resistance. The in-service coatings, however, are in most cases vulnerable to surface cracking since this dissimilar material pair responds differently to the thermal transients [1]. Once the cracking, driven by thermal stresses, penetrates the coating-substrate interface, interfacial delamination may occur subsequently due to the edge effects [2]. This situation poses a serious threat to the life of the coated components, since it will cause the final spallation of coating. Therefore, reliable performance of this thermo-mechanical system depends highly on coating's thermal shock resistance.

To enhance the coating's thermal shock resistance, the central issue is to delay the surface crack growth, which requires the calculation of the cracking driving force for the given thermal transients. Many investigations have been carried out to establish relationship between the cracking driving force and system parameters such as thermal conditions, crack depth as well as thermo-physical properties of the coating and substrate [3-8]. The derived results were then used as theoretical basis for various processing techniques to improve the integrity of coating-substrate system. To allow for misfit strain tolerance during thermal cycling, the microstructure of the ceramic top coat in Thermal Barrier Coatings (TBCs) was by design porous or columnar [9]. For the same purpose, a variety of inhomogeneous coating designs have been proposed, such as functionally gradient coating, duplex coating as well as multilayer coating [10-12].

Recently a new concept of coating design, which is in contrast to the aforementioned ideas of an intact coating, has been developed to enhance the durability of coating in a fluctuating thermal environment [13-15]. In this so called "segmented coating", the coating was intentionally introduced multiple segmentation cracks, which run from the surface of coating toward the interface of coating/substrate. It should be noted that the spacing of the formed segmentation cracks can be controlled by adjusting the processing parameters of hybrid technique of laser discrete pre-quenching substrate plus post-deposition, the details of which can be found elsewhere [15]. Our experimental 
observations [15] demonstrated that the segmented coating could largely survive the intact coating, i.e., the former can bear more thermal cycles prior to failure. In fact, the same beneficial effect of such a pre-cracked coating has also been reported for plasma sprayed TBCs by other research group [16-19]. Guo et al. [19] argued that segmentation cracks could enable coatings to tolerate greater strains, thereby improving thermal shock behaviors of coatings. Our purpose in the present work is to reveal the underlying mechanism of the above-mentioned beneficial effect of such a segmented coating from the standpoint of fracture mechanics.

The geometry under investigation is an outer-surface-coated hollow cylinder and the segmentation cracks within the segmented coating are modeled as a periodic array of axial edge cracks. Although for a finitely long cylinder the stress analyses are usually complicated 3-D problems and a crack may propagate in axial, circumferential or thickness direction, the problem is modeled here as a plane strain problem. This simplification is considered to represent a reasonable approximation to provide an upper bound solution [4]. Also for the sake of simplicity, both coating and substrate materials are assumed to be homogeneous, isotropic and linear-elastic, the thermo-mechanical constants are temperature-independent, and the thermo-elastic coupling effects and inertia effects are negligible. Previous literature on dynamic thermo-elasticity seemed to validate the last assumption [20, 21].

The paper is organized as follows. Problem formulation was presented in Section 2. In this Section, the transient temperature and thermal stress field for the corresponding crack-free hollow cylinder were also established. In Section 3, based on the assumed linearity of the materials, the principle of superposition was adopted. The solution of the multiple segmentation crack problem has been obtained by utilizing the calculated transient thermal stresses in Section 2 with opposite sign as the only crack surface traction. The transient thermal stress intensity factors (TSIFs) were evaluated using finite element method. Subsequently, the numerical results for the TSIFs were obtained in Section 3 as a function of normalized quantities such as time, convection severity, and segmentation crack spacing, crack depth. Finally, main conclusions of the present investigation were drawn in Section 4.

\section{Problem formulation}

As shown in Fig. 1, the considered substrate hollow cylinder (material \# 2) has an inner radius $a$ and an outer radius $b$, with the origin of cylindrical coordinates $(r, \phi, z)$ at its center.

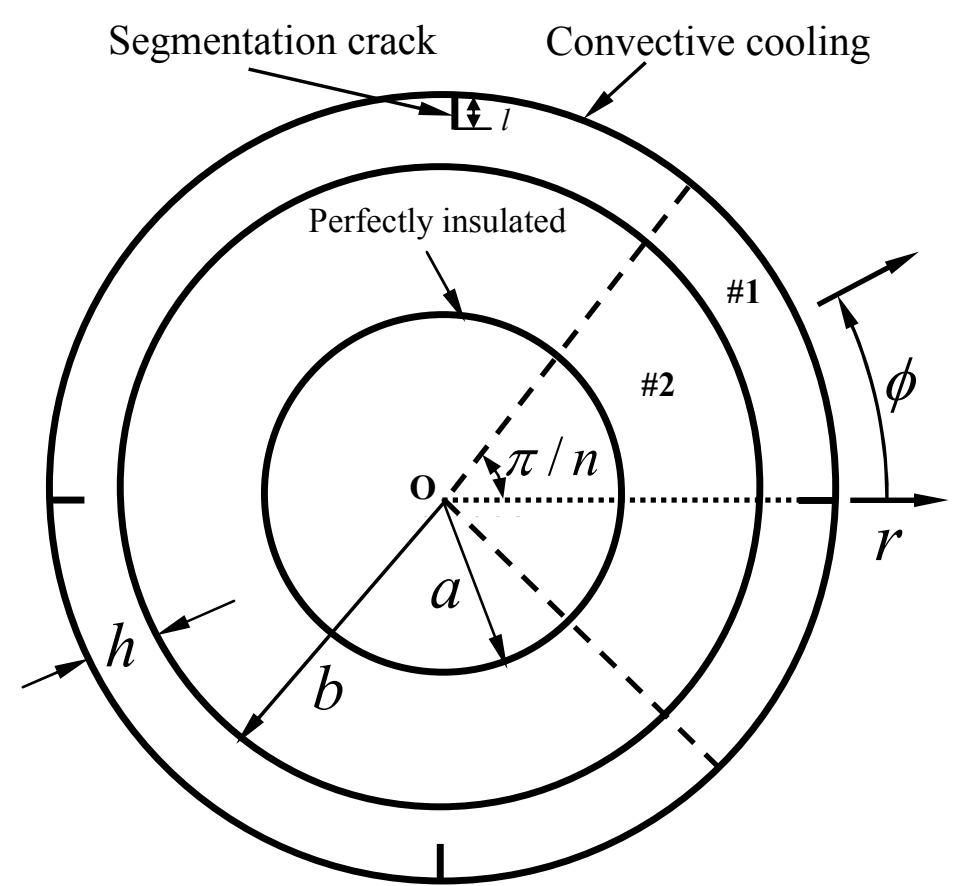

Fig.1. Schematic diagram of the segmented coating-substrate system subjected to convective cooling from outer surface. 
The segmented coating (material \# 1) of thickness $h$ contains $n$ uniformly spaced, radially oriented segmentation cracks of depth $l$ (Only four segmentation cracks are depicted in Fig.1.). The coated cylinder is assumed to be exposed to convective cooling from the outer surface, with the heat transfer coefficient $H$ and the ambient temperature $T_{a}$ while the inner surface is perfectly insulated.

\section{Temperature and thermal stress field for the crack-free hollow cylinder}

Let the temperature in the coating and substrate be $T_{1}$ and $T_{2}$, respectively. Since the crack-free cylinder is axisymmetric, there is no variation of temperature in the circumferential direction. Therefore, $T_{1}$ and $T_{2}$ would be functions of coordinate in radial direction $r$ and the time $t$ only. The temperature deviations $\theta_{i}(r, t)$ are defined by

$\theta_{i}(r, t)=T_{i}(r, t)-T_{0} \quad(i=1,2)$

Here, the subscripts 1 and 2 refer to coating and substrate, respectively. $T_{0}$ is the initial homogeneous temperature of the coated cylinder.

To find the temperature deviations $\theta_{i}(r, t)$ in the crack-free hollow cylinder, the following diffusion equation should be solved

$\frac{\partial^{2} \theta_{i}}{\partial r^{2}}+\frac{1}{r} \frac{\partial \theta_{i}}{\partial r}=\frac{1}{D_{i}} \frac{\partial \theta_{i}}{\partial t}$

The initial conditions are given by

$\theta_{2}(r, 0)=0(a \leq r \leq b) ; \quad \theta_{1}(r, 0)=0(b \leq r \leq b+h)$

The continuity and boundary conditions are written as

$\theta_{1}(b, t)=\theta_{2}(b, t), k_{1} \frac{\partial}{\partial r} \theta_{1}(b, t)=k_{2} \frac{\partial}{\partial r} \theta_{2}(b, t) \quad(t>0)$
$\frac{\partial}{\partial t} \theta_{2}(a, t)=0, \quad \frac{\partial}{\partial r} \theta_{1}(b+h, t)=\frac{H}{k_{1}}\left[\theta_{0}-\theta_{1}(a, t)\right] \quad(t>0)$

In above formula, $D=k / \rho c$ is the thermal diffusivity with $k$ the material thermal conductivity, $\rho$ the density and $c$ the specific heat. $\theta_{0}=T_{a}-T_{0}$, in the case of convective cooling, $\theta_{0}<0$. From Eq.(3b), the temperature and heat flux are assumed to be continuous at the bonded interface, indicating perfect interface conditions. The governing equation has been solved by using the standard Laplace transform technique as done in [22].

Since the segmentation cracks are assumed in the radial direction, the circumferential stresses $\sigma_{\phi}$ in the axisymmetric crack-free hollow cylinder are of primary interest because they determine the propagation of the crack in mode I fracture. The relevant displacement and stress components for the problem, under the assumption of plane strain conditions, can be expressed as [4]:

$$
\begin{aligned}
& u_{r}^{i}(r, t)=\frac{1+v_{i}}{1-v_{i}} \frac{\alpha_{i}}{r} \int_{a_{i}}^{r} \theta_{i}(\rho, t) \rho d \rho+C_{1 i} r+\frac{C_{2 i}}{r} \\
& \sigma_{r}^{i}(r, t)=-\frac{\alpha_{i} E_{i}}{1-v_{i}} \frac{1}{r^{2}} \int_{a_{i}}^{r} \theta_{i}(\rho, t) \rho d \rho+\frac{E_{i}}{1+v_{i}}\left(\frac{C_{1 i}}{1-2 v_{i}}-\frac{C_{2 i}}{r^{2}}\right) \\
& \sigma_{\phi}^{i}(r, t)=\frac{\alpha_{i} E_{i}}{1-v_{i}} \frac{1}{r^{2}} \int_{a_{i}}^{r} \theta_{i}(\rho, t) \rho d \rho-\frac{\alpha_{i} E_{i}}{1-v_{i}} \theta_{i}(r, t)+\frac{E_{i}}{1+v_{i}}\left(\frac{C_{1 i}}{1-2 v_{i}}+\frac{C_{2 i}}{r^{2}}\right)
\end{aligned}
$$

where $i=1,2$, and $a_{i}=b$ for $\mathrm{i}=1 ; a_{i}=a$ for $\mathrm{i}=2$. The symbols $\alpha, E, v$ denote coefficient of thermal expansion (CTE), Young's modulus, and Poisson's ratio, respectively.

Both the inner surface and outer surface are stress-free and at the interface, the displacement meets the requirement of continuity and the normal stresses are identical for the coating and substrate. 
Therefore, the unknown constants $C_{1 i}, C_{2 i}$ were determined from the following boundary and continuity conditions

$$
\begin{aligned}
& \sigma_{r 1}(a, t)=0 ; \quad \sigma_{r 2}(b+h, t)=0 \quad(t>0) \\
& u_{r 1}(b, t)=u_{r 2}(b, t) ; \sigma_{r 1}(b, t)=\sigma_{r 2}(b, t) \quad(t>0)
\end{aligned}
$$

\section{. Cracking analysis and thermal stress intensity factors}

As the result of convective cooling, at any time instant the stress intensity builds up around the segmentation crack tip. Taking advantage of the assumed linearity of the materials, the principle of superposition is adopted. The thermal stress problem for a coated hollow cylinder containing multiple segmentation cracks may now be solved by using the equal and opposite of the circumferential stress given by Eq.(4) as the crack surface traction. In the case of $n$ segmentation cracks evenly spaced and of equal depth $l$, only a representative sector with a central angle of $\pi / n$, has to be considered. Since the above cracking problem (transient mixed boundary problem) is analytically intractable and therefore will be solved using the FE method through commercial package ANSYS [23]. In order to pick up the square root singularity nature of the crack-tip strain field with high accuracy, the quarter points and singular elements were adopted near the crack tip. The detailed mesh configuration is schematically illustrated in Fig.2, in which quarter points and singular elements are labeled as Q and $\mathrm{S}$, respectively.

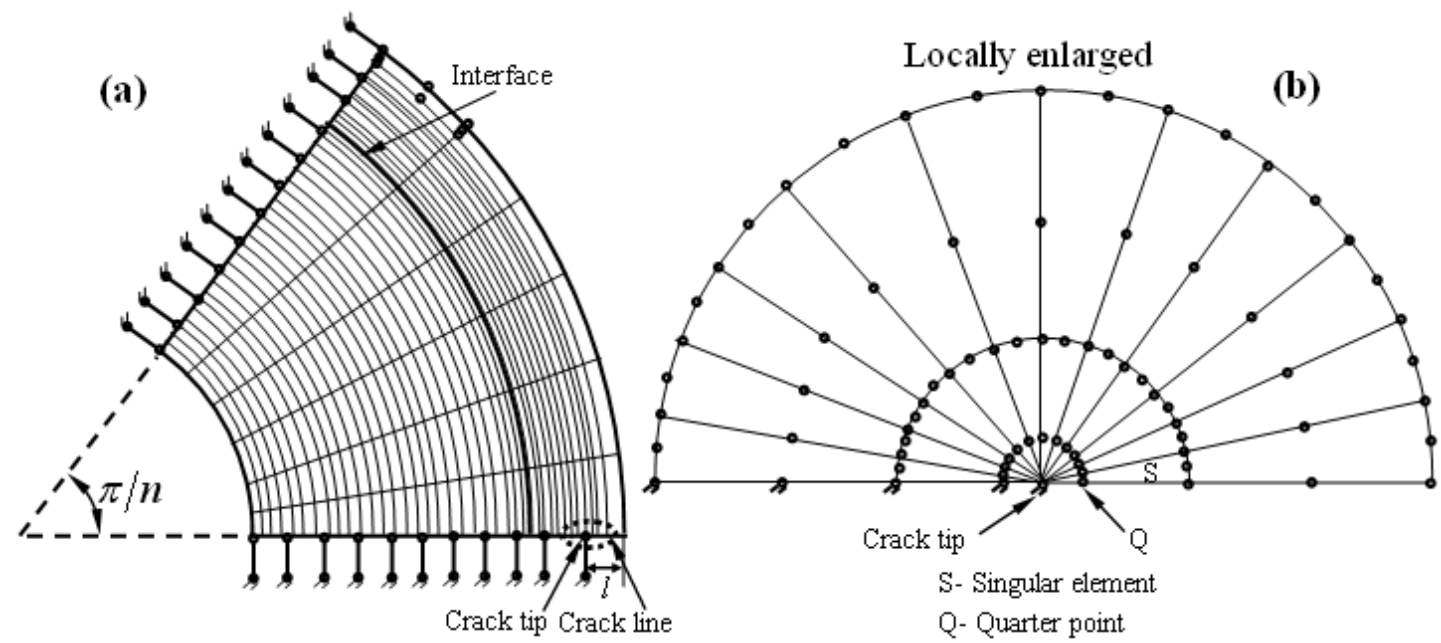

Fig.2. (a) Schematic diagram of a meshed representative sector with central angle of $\pi / n$.

The pseudo-traction upon the segmentation crack surface has the equal value of thermal stress from the crack-free problem, given by Eq.(4) but with an opposite sign. (b) Locally enlarged meshes configuration around the segmentation crack tip. The quarter points and the singular elements surrounding the crack tip are labeled as Q and S, respectively.

The driving force for the propagation of segmentation crack, represented by the Thermal Stress Intensity Factor (TSIF), is extracted from the FE solution which reads [24]:

$$
K_{\mathrm{I}}=\frac{E_{i} u_{\phi}}{\left(1+v_{\mathrm{i}}\right)\left(1+\kappa_{\mathrm{i}}\right)} \sqrt{\frac{2 \pi}{r_{q}}}
$$

Here, $u_{\phi}$ is the circumferential displacement at the quarter point of the singular element on the free face of the crack, $r_{q}$ is the distance from quarter point to the crack tip, $i=1$ when the crack tip is within the coating while $i=2$ within the substrate and $\kappa_{i}=3-4 v_{i}$ in the case of plane strain problem. 


\section{Results and discussions}

As an example for the thermal shock induced cracking problem of a segmented coating, a material pair corresponding to a ceramic coating and a stainless steel substrate, has been considered. The main dimensionless data concerning material properties and geometrical parameters in our calculations are tabulated in Table 1.

Table 1. The dimensionless quantities used in the analysis

\begin{tabular}{lllllll}
\hline$D_{2} / D_{1}$ & $k_{2} / k_{1}$ & $E_{2} / E_{1}$ & $v_{2} / v_{1}$ & $\alpha_{2} / \alpha_{1}$ & $a / h$ & $b / h$ \\
\hline 1.370 & 1.582 & 3.155 & 0.940 & 1.644 & 50 & 100 \\
\hline
\end{tabular}

\section{Selected results for the temperature and stress field in crack-free cylinders}

The normalized temperature deviation in a crack-free hollow cylinder, $\theta / \theta_{0}$, is plotted against normalized radial coordinate in Fig. 3 for various dimensionless time, $\mathrm{Fo}=100,500,1000$, and under different convection severity, $\mathrm{Bi}=0.1$ and $\mathrm{Bi}=\infty$ (thermal shock case). Here, the dimensionless time and convection severity are defined as $\mathrm{Fo}_{2}=D_{1} t / h^{2}$ and $\mathrm{Bi}=H h / k_{1}$, respectively. It is shown that at a certain time instant, the normalized temperature deviation, $\theta / \theta_{0}$, increases as the distance from the inner surface increases. At a fixed location, the normalized temperature deviation also increases with the elapse of time. As time goes to infinity, the normalized temperature deviation reaches the limit of unity, which is approached much faster for greater values of Bi.

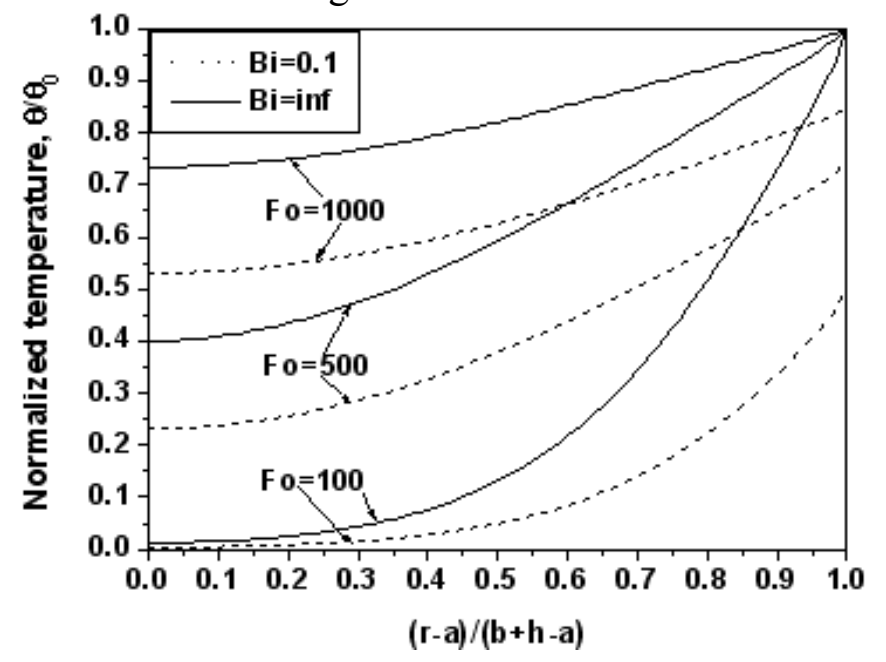

Fig.3. The normalized temperature deviation versus normalized radial coordinate for various dimensionless time instants and under different convection severity. "inf" stands for infinity, and other inputs are listed in Table 1.

In Fig.4, the variation of the normalized circumferential stress in a crack-free cylinder versus normalized radial coordinate is presented for different normalized time, Fo $=0.1,10,50,100$. In this plot, the convection severity is taken as infinity and $\sigma_{0}=-E_{1} \alpha_{1} \theta_{0} /\left(1-v_{1}\right)$. It can be seen that the tensile circumferential stress distribution within coating is at most time instants non-uniform, especially for small time instant. In terms of the differences in thermo-physical properties of coating and substrate, circumferential stress discontinues at the interface as expected. Due to the self-equilibrating nature of thermal stress, there should exist a compressive stress region somewhere near the inner surface (not shown here). The peak value of the thermal stress at the outer surface, which occurs at $t=0$, has the expected value of $\sigma_{0}$. This is the thermal stress in a fully constrained plate undergoing a sudden uniform temperature change $\theta_{0}$. It can also be seen that at a fixed location, the normalized circumferential stress within coating first increases, then decreases with elapse of time. However, the stress gradient within coating decreases steadily with time. 


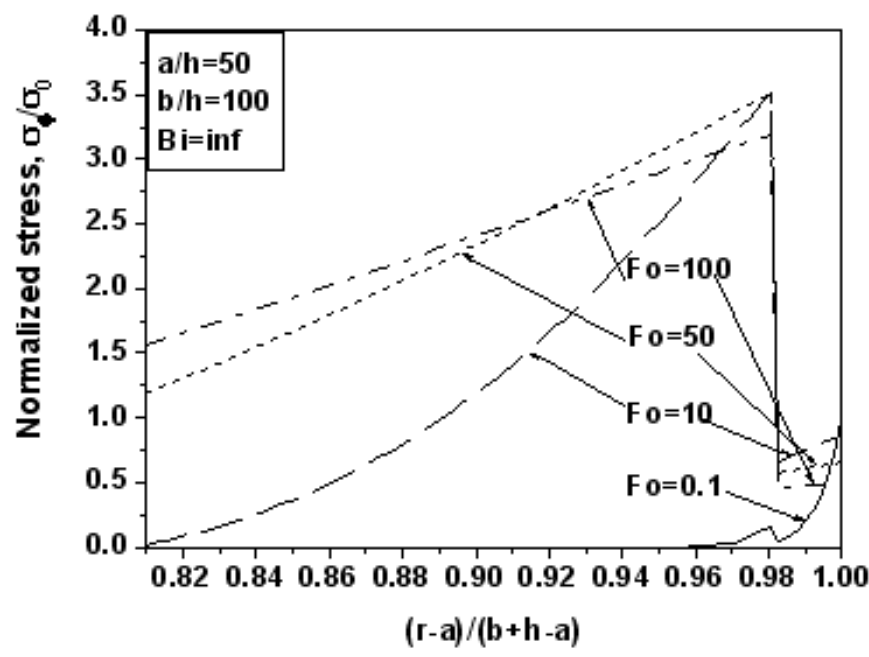

Fig.4. The normalized circumferential stress versus normalized radial coordinate for various time instants. "inf" stands for infinity and other inputs are listed in Table 1.

\section{Selected results for the thermal stress intensity factors for segmentation cracks}

Fig.5 shows the normalized TSIF versus normalized time for various normalized segmentation crack depth and under different convection severity. In this graph, the number of segmentation crack is taken to be thirty and $K_{0}=-E_{1} \alpha_{1} \theta_{0} \sqrt{h} /\left(1-v_{1}\right)$. It can be seen that, with the elapse of time, the normalized TSIF first increases, goes through a maximum, and then decreases. This variation trend may be explained by the change in the stress profile with increasing time in Fig.4. In the case of thermal shock $(\mathrm{Bi}=\infty)$, although the peak circumferential thermal stress in a crack-free cylinder occurs at time $t=0$ (see Fig.4), the peak value of TSIF is reached at some time after the thermal shock. Therefore, based on the strength criteria the initiation of coating cracking occurs at the very beginning of a thermal shock, while based on the fracture toughness criteria the maximum driving force for cracking extension occurs after some delay time. This delay time is very short for very small crack depths and increases with increasing crack depth. Another indication of this graph is that, the most dangerous instant the coating experience is not at, but before the steady state of thermal transients. Therefore, the damage tolerance analysis performed only at the steady state of thermal transients will obviously underestimate its severity in practical situation. The influence of convection severity, measured by $\mathrm{Bi}$, on the TSIF can also be induced from Fig.5. As more docile severe thermal convection is applied, not only does the magnitude of peak TSIF become lower, but also the peak value occurs later.

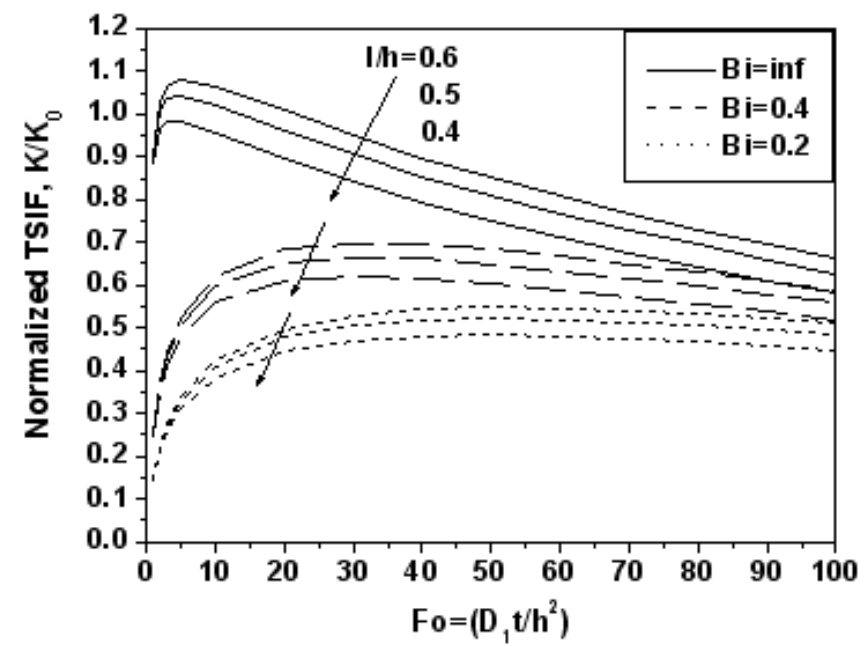

Fig.5. The normalized TSIF versus normalized time for various normalized segmentation crack depth and under different convection severity. "inf" stands for infinity, $n=30$ and other inputs are listed in Table 1. 
The variation of normalized TSIF versus normalized segmentation crack spacing for various segmentation crack depth and under different convection severity is depicted in Fig.6. Here, the dimensionless time instant, Fo, is taken to be fifty and $s=2 \pi(b+h) / n$, is the segmentation crack spacing. It is shown that, as the segmentation crack spacing decreases, the magnitude of TSIF first almost plateaus; when the segmentation crack spacing is smaller than a critical value, the decrease in TSIF with decreasing crack spacing (i.e. increasing crack density) is very significant, especially for large values of crack depth. Approximately, a single segmentation crack gives rise to the maximum TSIF for all possible crack depths. This interesting and important phenomenon is commonly referred to as "load relief effect" [25] or "shielding effect" [26, 27] for the interaction of parallel cracks. The present investigation confirms the effectiveness of this beneficial effect for the case of thermal shock condition. Hence, the reason why a segmented coating has higher durability than an intact counterpart is that the former can cause the drop of crack driving force for segmentation crack propagation. It should be emphasized that, only until the segmentation crack spacing is narrow enough can this beneficial effect be shown remarkably. It is expected that this result may rationalize the newly developed hybrid processing technique [13-15], leading to the enhancement of coating's thermal shock resistance by multiple segmentation cracks.

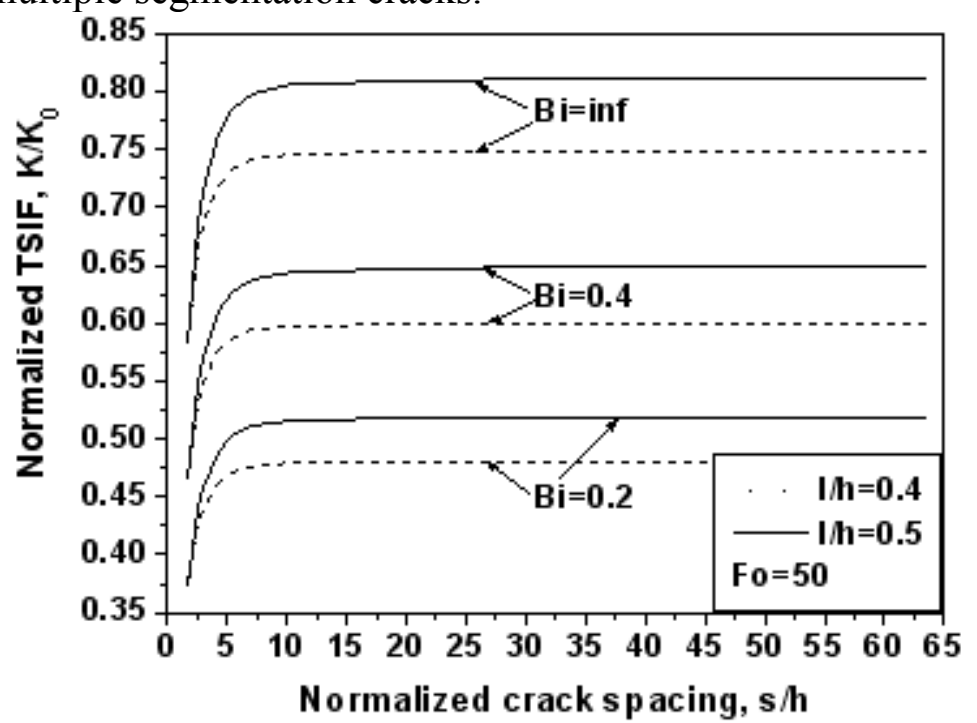

Fig.6. The normalized TSIF versus normalized segmentation crack spacing for various normalized crack depth and under different convection severity. "inf" stands for infinity. Fo=50 and other inputs are listed in Table 1.

\section{Conclusions}

In this work, the thermal shock induced driving force for segmentation crack propagation within a segmented coating has been investigated. The peak TSIF has been found to occur neither at the beginning nor at the steady state of thermal transients, but at an intermediate instant. The dependence of TSIF on the segmentation crack spacing provides a quantitative mechanistic understanding of the experimentally observed beneficial effect of a segmented coating, which demonstrates much higher thermal shock resistance than an intact counterpart, if only the segmentation crack spacing is narrow enough.

\section{Acknowledgements}

The authors gratefully acknowledge the financial support of the National Natural Science Foundation of China (Grant No. 10802007) and the Specialized Research Fund for the Doctoral Program of Higher Education of Ministry of Education (Grant No. 20070008003). 


\section{References}

[1] J.W. Hutchinson, Z. Suo, ASME Adv. Appl. Mech. 29 (1992) p.63

[2] H.H. Yu, M.Y. He, J.W. Hutchinson, Acta Mater. 49 (2001) p.93

[3] H.F. Nied, Eng. Fract. Mech. 20 (1984) p.113

[4] R. Tang, F. Erdogan, ASME J. Eng. Gas. Turb. Power 107 (1985) p.212

[5] F. Erdogan, A.A. Rizk, Int. J. Fract. 53 (1992) p.159

[6] C.K. Chen, B.L. Kuo, Eng. Fract. Mech. 49 (1994) p.381.

[7] X. Chen, K. Zhang, G. Chen, G. Luo, Int. J. Solids Struct. 43 (2006) p.6424

[8] A.A. Rizk, Theor. Appl. Fract. Mech. 49 (2008) p.251

[9] J. Yan, T. Leist, M. Bartsch, A.M. Karlsson, Acta Mater. 56 (2008) p.4080

[10] J.H. Kim, M.C. Kim, C.G. Park, Surf. Coat. Technol. 168 (2003) p.275

[11] J.L. He, K.C. Chen, Surf. Coat. Technol. 200 (2005) p.1464

[12] Y.Z. Tsai, J.G. Duh, Surf. Coat. Technol. 200 (2005) p.1683

[13] Y. Hu, K. Zhang, G.N. Chen, C.W. Wu, J. Metal Treatment (Suppl.) 30 (2005) p.161(in Chinese)

[14] K. Zhang, C.W. Wu, Y. Hu, G.N. Chen, Solid State Phenomena 118 (2006) p243.

[15] G.N. Chen, G.X. Luo, K Zhang, X.Y. Xu, H. Shen, H.M. Yan, D.S. Rao, Acta Armament (Suppl.) 24 (2003) p.6. (in Chinese)

[16] B. Zhou, K. Kokini, Surf. Coat. Technol. 187 (2004) p.17

[17] B. Zhou, K. Kokiki, Acta Mater, 52 (2004) p.4189

[18] H.B. Guo, R. Vaben, D. Stover, Surf. Coat. Technol. 186 (2004) p.353

[19] H.B. Guo, S. Kuroda, H. Murakami, Thin Solid Films, 506-507 (2006) p.136

[20] E. Sternberg, J.G. Chakravorty, ASME J. Appl. Mech. 26 (1959) p.503

[21] T. Atarashi, S. Minagawa, Int. J. Eng. Sci. 30 (1992) p.1543

[22] H.S.. Carslaw, J.C. Jaeger, Conduction of Heat in Solids, Oxford University, Oxford, 1986

[23] Ansys: Guidance to structural analysis (V.0205), Ansys Inc., 2000

[24] P.P. Lynn, A.R. Ingraffea, Int. J. Num. Methods Eng. 12 (1978) p.1031

[25] D.P. Rooke, F.I. Baratta, D.J. Cartwright, Eng. Fract. Mech. 14 (1981) p.397

[26] G.W. Schulze, F. Erdogan, Int. J. Solids Struct. 35 (1998) p.3615

[27] A.P. Parker, Eng. Fract. Mech. 62 (1999) p.577 
Advanced Manufacturing Technology, ADME 2011

10.4028/www.scientific.net/AMR.314-316

Understanding the Enhancement of Coating's Thermal Cracking Resistance by Multiple Segmentation 10.4028/www.scientific.net/AMR.314-316.223 